CERN LIBRARIES, GENEVA

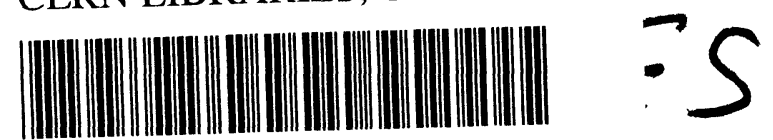

CM-P00057422

\title{
Spectral Functions in the Static Theory ${ }^{+}$
}

\author{
J. D. Walecka* \\ CERN - Geneva
}

\footnotetext{
+) Submitted to the Nuovo Cimento.

*) N.S.F. Postdoctoral Fellow.
} 
The spectral functions $G_{\mathrm{E}, \mathbb{M}}\left(\sigma^{2}\right)$ describing the electromagnetic properties of nucleons are related to the form factors $F_{E \cdot M}\left(q^{2}\right)$ by

$$
F_{E, M}\left(q^{2}\right)=\frac{1}{\pi} \int_{4 \mu^{2}}^{\infty} \frac{G_{E, M}\left(\sigma^{2}\right) d \sigma^{2}}{\sigma^{2}+q^{2}}
$$

where $q^{2}$ is the four-momentum transfer occuring at the electromagnetic vertex and $\mu=m_{\pi}$. The spectral functions can be regarded as the quantities of fundamental theoretical interest and there have been two recent attempts at the calculation of these quantities using dispersion theory, one by Chew, Karplus, Gasiorowicz and Zachariason and the other by Federbush, Goldberger and Treiman 1),2).

The purpose of this paper is two-fold. First it will be shown how one can calculate the spectral functions within the framework of the static theory and that the functions thus calculated agree with the static limit of the dispersion theory result. The validity of equation (1) has not yet been proved in the framework of relativistic field theory. Moreover, the computation of the spectral functions mentioned above are based on the analytic continuation of the pionnucleon scattering amplitude for unphysical values of the angle. Therefore, it is thought useful to associate with these calculations a straightforward computation based on a simple model. 
The advantage of dealing directly with the spectral functions instead of the form factors in the static theory is that in the no cut-off limit the former are finite whereas the latter are not. Now it might be hoped that the static theory would at least explain the low $\sigma^{2}$ part of the spectral functions, but the work of references 1) and 2) has shown that the discrepancy between dispersion theory results and their static limit are great and that they persist even down to $\left.\sigma^{2}=4 \mu^{2} 3\right)$. Therefore, the second problem dealt with here will be the result of including the $\frac{1}{M}$ nucleon recoil corrections into the framework of the static model and showing:

a) the presence of these purely kinematical corrections profoundly alter the spectral function.

b) The kinematical $\frac{1}{\mathbb{M}}$ corrections are in good agreement with the $\frac{1}{\mathbb{M}}$ corrections calculated by dispersion theory techniques indicating that this is the main source of difference between the two theories.

The first conclusion indicates that one can have little faith in the predictions of the static theory in the electromagnetic structure problem. Also, since the partial waves of higher $\ell$ value (the scattering in these states is known neither experimentally, nor theoretically), become very important in the calculation of $G_{\mathbb{E}, \mathbb{M}}\left(\sigma^{2}\right)$, there may be some doubt as to the validity of using only $S$ and $P$ waves in the analytic continuation of the meson-nucleon scattering amplitude as is done in the dispersion theory approach. 
The calculation of the electromagnetic properties of the nucleon in the static theory has been discussed by several authors 4 ). In this work the notation of the last author (F) shall be used. The introduction of nucleon recoil is based on the work of Bosco, Fubini and Stanghellini 5), who use the procedure to reproduce, up through D waves, the meson-nucleon scattering phase shifts calculated from dispersion theory.

In section II, the working equations are derived. In section III the spectral functions are calculated and in section IV they are related to the corresponding quantities from the relativistic theory.

\section{Derivation of equations.}

In the following, the Breit frame will be used, that is, the initial nucleon momentum is $-\frac{\vec{q}}{2}$ and the final nucleon momentum is $+\frac{\vec{q}}{2}$ where $\vec{q}$ is the momentum of the photon from the external field. In this system there is no energy transferred to the nucleon and hence $q^{2}=\vec{q}^{2}$ and al so there is only one momentum vector, $\vec{q}$ for constructing invariants. Now

$$
H\left| \pm \frac{\vec{q}}{b_{2}}\right\rangle=E_{\frac{q_{2}}{\sigma_{2}}}\left| \pm \frac{\vec{q}}{\sigma_{2}}\right\rangle=\left(M^{2}+\left(\frac{\vec{q}}{v_{2}}\right)^{2}\right)^{1 / 2}\left| \pm \frac{\vec{q}}{b_{2}}\right\rangle
$$

where $H$ is the total meson-nucleon Hamiltonian and $\left| \pm \frac{\vec{q}}{2}\right\rangle$ is a physical nucleon. One thus includes the proper kinematics by 
replacing $H$ by $E_{\frac{q}{2}}$ when acting on a physical nucleon state but then uses the static model for the dynamics and sums over states. The reason for this is that the dynamics of the static model are relatively simple. To calculate corrections rigorously to $\frac{1}{\mathbb{M}}$ one must also, of course, include $\frac{1}{\mathbb{M}}$ modifications of the dynamics, or $\mathrm{H}$ but this complicates matters and it will be shown that the kinematical corrections alone are sufficient to give $\frac{1}{\bar{M}}$ corrections similar to those of dispersion theory.

One starts from

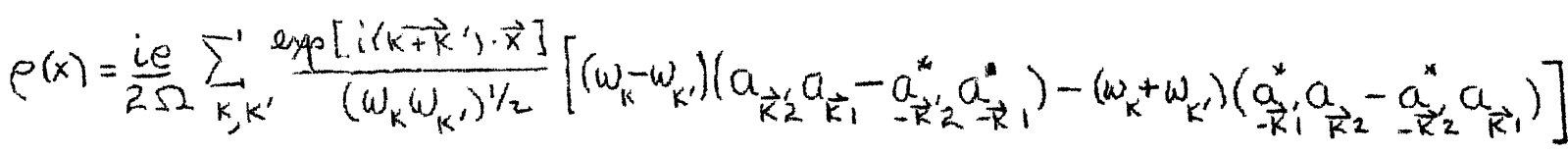

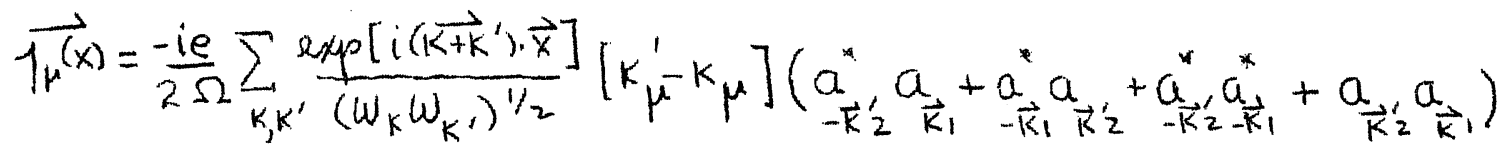

which are the expressions for the charge and current densities in the meson field. The subscripts 1 and 2 refer to the isotopic spin of the Hermitian charged fields. What one wants to calculate is the transition probability:

$$
\omega=\left\langle\vec{q}_{2}\left|\int d^{3} x\left(e^{(x)} \vec{\phi}^{\text {ent }}(x)-\overrightarrow{j(x)} \cdot \vec{A}^{\text {ent }}(x)\right) \cdot\right|-\frac{\vec{q}}{2}\right\rangle
$$

where one takes

$$
\begin{aligned}
& \Phi^{\text {ant }}(x)=\phi e^{i \vec{q} \cdot \vec{x}} \\
& \vec{A}^{\text {ext }}(x)=\vec{a} e^{i \vec{g} \cdot \vec{x}}
\end{aligned}
$$


5 .

and defines (using invariance properties):

$$
\omega=F_{E}\left(\vec{q}^{2}\right) \phi-i \vec{\sigma} \cdot\left(\vec{q}_{\wedge} \vec{a}\right) F_{M}\left(\vec{q}^{2}\right)
$$

The f's are the form factors in the static theory. The procedure is to relate the expectation value of pairs of creation and annihilation operators to the meson nucleon scattering amplitudes $(F) . \quad a_{\vec{k} \alpha}^{*} a_{\vec{k} \alpha^{\prime}}$ shall be used as an example in the following and then the results given for all pairs, starting from

$$
\begin{gathered}
H=H_{0}+H_{1} \\
{\left[H_{0}, a_{\vec{k} \alpha}^{*}\right]=\omega_{k} a_{\vec{k} \alpha}^{*}} \\
{\left[H, a_{\vec{k} \alpha}^{*}\right] \equiv V_{\vec{k} \alpha}} \\
\left\langle\frac{\vec{q}}{2}\left|\left[H, a_{\vec{k} \alpha}^{*} a_{\vec{k}^{\prime} \alpha^{\prime}}\right]\right|-\frac{\vec{q}}{2}\right\rangle=0
\end{gathered}
$$

$$
\begin{aligned}
& \text { one finds }
\end{aligned}
$$

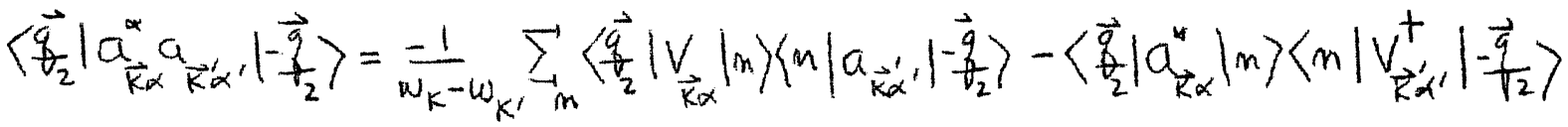

$$
\begin{aligned}
& \text { Taking matrix elements of the following relations ( } \vec{P} \text { is the total } \\
& \text { momentum operator) } \\
& {\left[\vec{p}, a_{\vec{k} \alpha}^{*}\right]=\vec{k} a_{\vec{k} \alpha}^{*}} \\
& {\left[\vec{p}, a_{\vec{k} \alpha}^{\alpha} a_{\vec{k}^{\prime} \alpha^{\prime}}\right]=\left(\overrightarrow{k-k^{\prime}}\right) a_{\vec{k} \alpha}^{*} a_{\vec{k}^{\prime} \alpha^{\prime}}} \\
& \text { one gets } \left.\quad\left(P_{m}-P_{m}-k\right)<m\left|a_{\vec{k} \alpha}^{n}\right| n\right\rangle=0 \\
& \left.\left(\overrightarrow{P_{m n}}-P_{m}+k\right)<m\left|a_{\vec{k} \alpha}\right| n\right\rangle=0 \\
& \left(P_{m}-P_{m}-k+k^{\prime}\right)\left\langle m\left|a_{\vec{k} \alpha}^{*} a_{\vec{k}^{\prime} \alpha^{\prime}}\right| n\right\rangle=0
\end{aligned}
$$

5907 
expressing the conservation of momentum.

\section{Also from}

$$
\left[H, a_{\vec{k} \alpha}\right]\left| \pm \overrightarrow{q_{2}}\right\rangle=\left(H-E_{q / 2}\right) a_{\vec{k} \alpha}\left| \pm \overrightarrow{g_{2}}\right\rangle
$$

one has

$$
a_{\vec{k} \alpha}\left| \pm \frac{\vec{G}}{D_{2}}\right\rangle=\frac{-1}{H+w_{k}-E_{q / 2}} V_{\vec{k} \alpha}^{+}\left| \pm \frac{\vec{g}}{b_{2}}\right\rangle
$$

Therefore

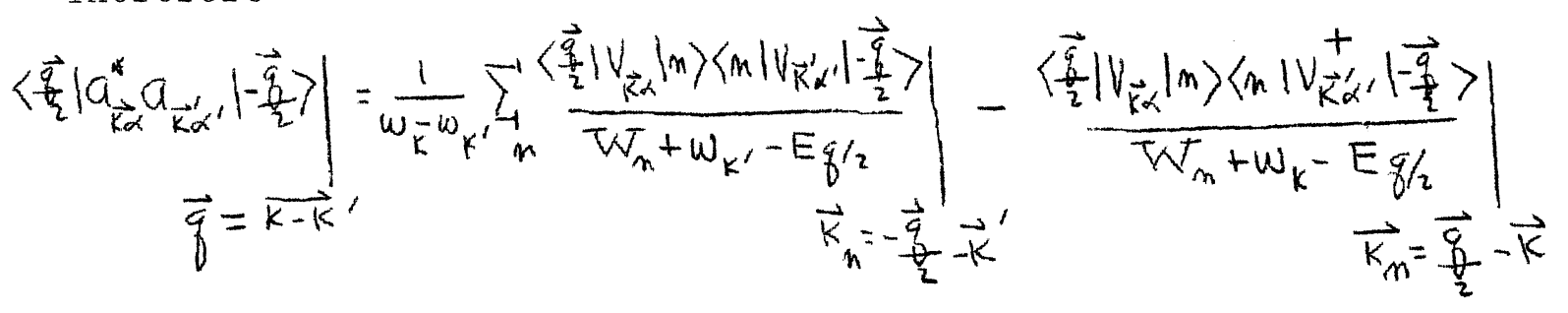

$W_{n}$ us the energy of the intermediate state $n$. Using

$$
W_{m}=\left(M_{m}^{2}+k_{m}^{2}\right)^{1 / 2}
$$

and

$$
\omega_{n} \equiv W_{n}-M
$$

one finds to order $\frac{1}{M} \quad\left(i . e\right.$. if $\left.\frac{\omega_{m}}{M} \ll 1\right)$

$$
W_{m}-E_{g / 2}=w_{n}+\frac{\vec{k} \cdot \vec{k}}{2 M}
$$

Until now the derivation has been rigorous. One now uses the static model to evaluate the numerators. Replacing $\left| \pm \overrightarrow{\frac{9}{2}}\right\rangle \rightarrow|\mathrm{R}\rangle$ where $|R\rangle$ denotes one of the four physical nucleon spin states 
7 .

$$
\begin{aligned}
& \text { one has }
\end{aligned}
$$

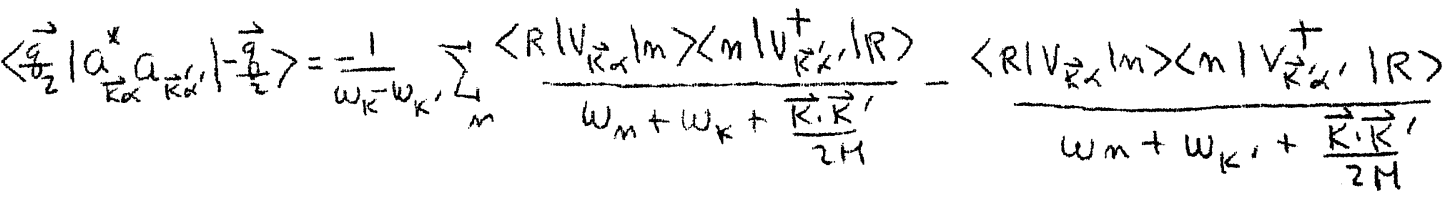

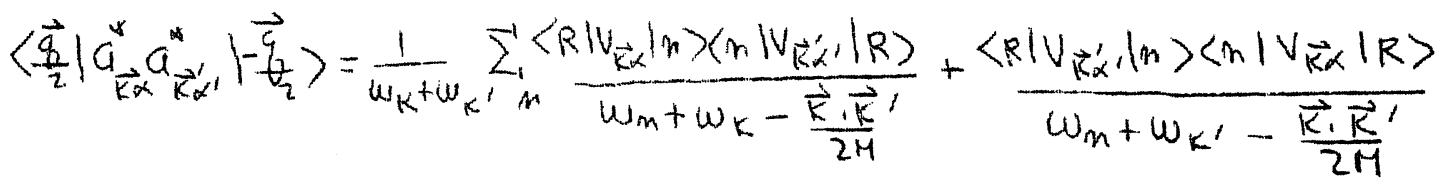

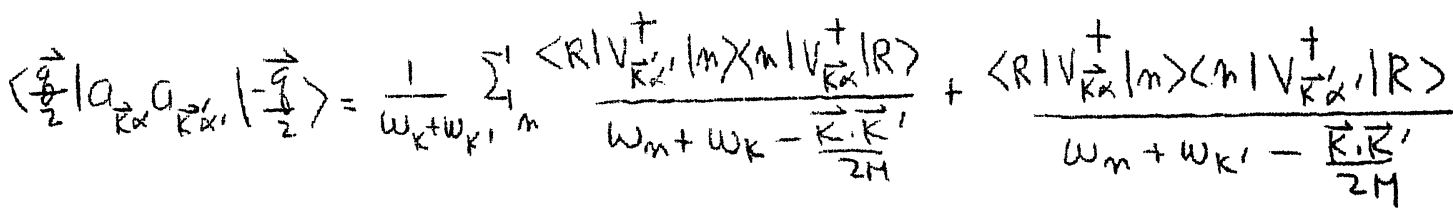

where

$$
V_{\vec{k} \alpha}=i\left(\frac{f^{0}}{\mu}\right)\left(\frac{4 \pi}{2 w_{k}}\right)^{1 / 2}(\vec{\sigma} \cdot \vec{k}) \tau_{\alpha} V(k)
$$

Notice the only difference from $(F)$ is the extra term in the denominators. Proceeding as in (F), one writes the unitarity principle

$$
\frac{3 i}{2 p^{3} v^{2}(p)}\left\langle i \alpha_{j}\left|T^{\dagger}-T\right| j \alpha^{\prime} ; r^{\prime}\right\rangle=\left(\frac{f_{0}}{\mu}\right)^{2} \sum_{n} \delta\left(\omega_{n}-\omega_{p}\right)\left(R\left|\sigma_{i} \tau_{\alpha}\right| m-\right)\left(n-\left|\sigma_{j} \tau_{\alpha},\right| R\right)
$$

where $T$ is defined between free meson and bare spinor states and $\mathrm{n}$ - is an incoming scattering state. Multiplying this equation by

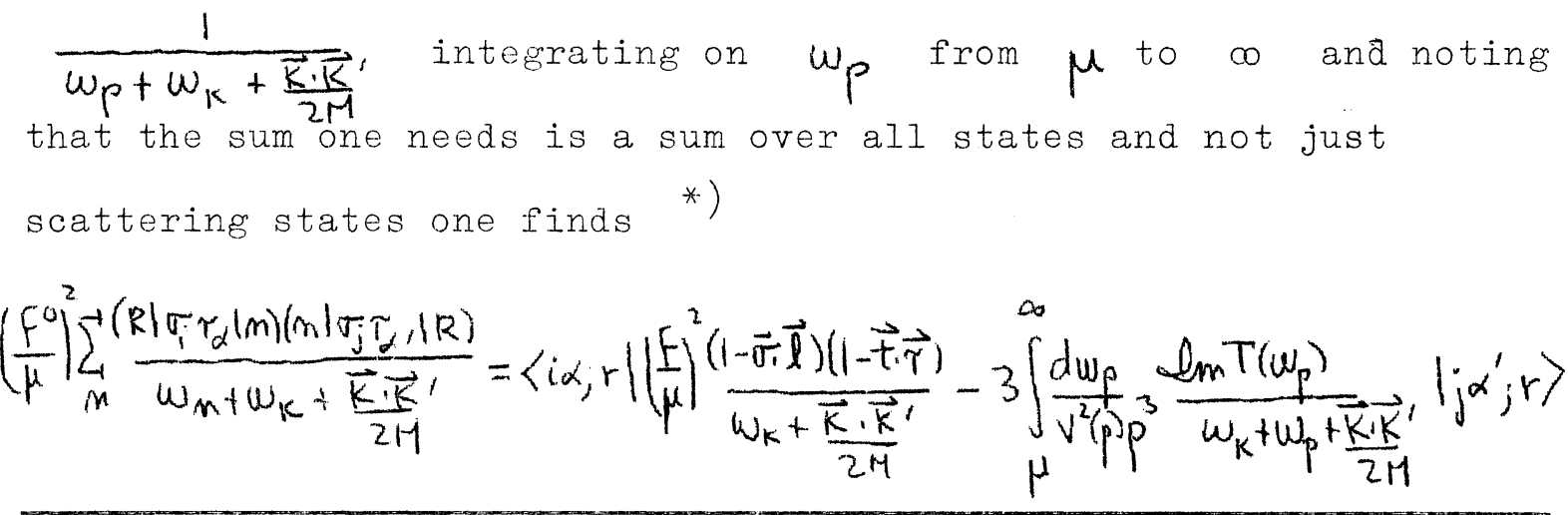

*) Note 6): $\left\langle i \alpha_{j} r|1-\vec{t} \cdot \vec{\tau}| \alpha_{j}^{\prime} r^{\prime}\right\rangle=\delta_{i j}\left\langle r\left|\tau_{\alpha} \tau_{\alpha}\right| r^{\prime}\right\rangle=\left\langle j \alpha^{\prime} ; r|1+\vec{t} \cdot \vec{\tau}| 1 \alpha ; r^{\prime}\right\rangle$

5907

$$
\left\langle i \alpha ; r|1-\vec{l} \cdot \vec{\sigma}| j \alpha^{\prime} ; r^{\prime}\right\rangle=\delta_{\alpha \alpha^{\prime}}\left\langle r\left|\sigma_{i} \sigma_{j}\right| r^{\prime}\right\rangle=\left\langle j \alpha^{\prime} ; r|1+\vec{\lambda} \cdot \vec{\sigma}| i \alpha ; r^{\prime}\right\rangle
$$


where $f$ is now the renormalized coupling constant. One now takes. into account the experimental evidence of the dominance of the 3-3 scattering resonance. Approximating the contribution of the resonance by a $\delta$ function one has

$$
-3 \int_{\mu}^{\infty} \frac{d w_{p}}{v^{2}(p) p^{3}} \frac{\operatorname{lm} T\left(w_{p}\right)}{w_{k}+w_{p}+\frac{\vec{k} \cdot \vec{k}^{\prime}}{2 H}}=\left(\frac{a}{\mu}\right)^{2} \frac{(2+\vec{t} \cdot \vec{\tau})(2+\vec{l} \cdot \vec{\tau})}{w_{k}+w_{R}+\frac{\vec{k} \cdot \vec{k}^{\prime}}{2 M}}
$$

with $a^{2}$ the effective strength $\left(a^{2}=\frac{4}{q} f^{2}\right.$ theoretically $)$ and $\omega_{R}$ the resonance energy $\left(\omega_{R} \approx 2 \mu\right)$ one finds

$$
\begin{aligned}
& \left(\frac{\vec{q}}{2}\left|a_{k_{\alpha^{\prime}}+\vec{k}^{\prime} \alpha^{\prime}}\right|-\frac{\vec{q}}{2}\right)=\sum_{i j}^{-1} \frac{c\left(k k^{\prime}\right)}{w_{k}-w_{k^{\prime}}} k_{i} k_{j}^{\prime}\left\langle i \alpha r\left|R\left(w_{k}\right)-R\left(w_{k^{\prime}}\right)\right| j \alpha^{\prime} r\right\rangle \\
& \left(\frac{\vec{q}}{2}\left|a_{\vec{k} \alpha}^{\alpha} a_{\vec{k}^{\prime} \alpha^{\prime}}^{\alpha}\right| \overrightarrow{\frac{q}{2}}\right)=-\sum_{i j} \frac{c\left(k k^{\prime}\right)}{w_{k}+w_{k}} k_{i} k_{j}^{\prime}\left\langle\alpha r\left|R\left(w_{k}\right)+\overline{R\left(w_{k}\right)}\right| j \alpha^{\prime} r\right\rangle \\
& \left(\frac{\vec{q}}{i}\left|a_{\vec{k} \alpha^{\prime}} a_{\vec{k}^{\prime} \alpha^{\prime}}\right|-\frac{\vec{q}}{2}\right)=-\sum_{i j} \frac{c\left(k k^{\prime}\right)}{w_{k}+w_{k^{\prime}}} k_{i} k_{j}^{\prime}\left\langle i \alpha r\left|\overrightarrow{R\left(w_{k}\right)}+R\left(w_{k^{\prime}}\right)\right| j \alpha^{\prime} r\right\rangle
\end{aligned}
$$

with

$$
c\left(k, k^{\prime}\right)=\frac{2 \pi v(k) v\left(k^{\prime}\right)}{\left(\omega_{k} \omega_{k 1}\right)^{1 / 2}}
$$

where

$$
\begin{aligned}
& R(\omega)=R_{1}(\omega)+R_{1}(\omega)(\vec{\sigma} \cdot \vec{l}+\vec{F} \cdot \vec{\tau})+R_{3}(\omega)(\vec{\sigma} \cdot \vec{l})(\vec{t} \cdot \vec{\tau}) \\
& \left\langle i \alpha r|\overline{R(\omega)}| j \alpha^{\prime} r^{\prime}\right\rangle \equiv\left\langle j \alpha^{\prime} r|R(\omega)| i \alpha r^{\prime}\right\rangle
\end{aligned}
$$




$$
\begin{aligned}
& R_{1}\left(\omega_{k}\right)=\left(\frac{F}{\mu}\right)^{2}\left(\frac{1}{\omega_{k}}+\frac{\vec{k} \cdot \vec{k}^{\prime}}{2 H \omega_{k}^{2}}\right)+4\left(\frac{a}{\mu}\right)^{2}\left(\frac{1}{\omega_{k}+\omega_{R}}+\frac{\vec{k} \cdot \vec{k}^{\prime}}{2 M\left(\omega_{k}+\omega_{R}\right)^{2}}\right) \\
& R_{2}\left(\omega_{k}\right)=-\left(\frac{f}{\mu}\right)^{2}\left(\frac{1}{\omega_{k}}+\frac{\vec{k} \cdot \vec{k}^{\prime}}{2 M \omega_{k}^{2}}\right)+2\left(\frac{a}{\mu}\right)^{2}\left(\frac{1}{\omega_{k}+\omega_{R}}+\frac{\vec{k} \cdot \vec{k}^{\prime}}{2 M\left(\omega_{k}+\omega_{R}\right)^{2}}\right) \\
& R_{3}\left(\omega_{k}\right)=\left(\frac{F}{\mu}\right)^{2}\left(\frac{1}{\omega_{k}}+\frac{\vec{k} \cdot \vec{k}^{\prime}}{2 \mu \omega_{k}^{2}}\right)+\left(\frac{a}{\mu}\right)^{2}\left(\frac{1}{\omega_{k}+\omega_{R}}+\frac{\vec{k} \cdot \vec{k}^{\prime}}{2 H\left(\omega_{k}+\omega_{R}\right)^{2}}\right)
\end{aligned}
$$

$R\left(\omega_{K^{\prime}}\right)$ is obtained from $R\left(\omega_{K}\right)$ by interchanging $\vec{K}$ and $\overrightarrow{K^{\prime}}$. substituting in the expressions for $\rho(x)$ and $\overrightarrow{j(x)}$ one has

$$
F_{E}\left(\vec{q}^{2}\right)=\frac{-e T_{3}}{\pi^{2}} \iint \frac{d^{3} k d^{3} k^{\prime} v(k) v\left(k^{\prime}\right)\left(\vec{k}^{\prime} \cdot \vec{k}^{\prime}\right) S\left(k^{\prime}+\vec{k}^{\prime}+\vec{q}^{\prime}\right)}{k^{2}-k^{\prime 2}}\left[R_{2}\left(w_{k}\right)-R_{2}\left(\omega_{k^{\prime}}\right)\right]
$$

$\left.-i \vec{\sigma} \cdot(\vec{q} \vec{a} \vec{a}) F_{H}\left(\vec{q}^{2}\right)=\frac{i e T_{3}}{\pi^{2}} \iint \frac{d^{3} k d^{3} k^{\prime} v(k) v\left(k^{\prime}\right)(\vec{k} \cdot \vec{a})\left(\vec{\sigma} \cdot\left(\vec{k}_{\mu} \vec{k}^{\prime}\right) S\left(\vec{k}+\vec{k}^{\prime}+\vec{q}\right)\right.}{k^{2}-k^{\prime 2}} \int \frac{R_{\frac{3}{2}}\left(\omega_{k}\right)}{\omega_{k}}-\frac{R_{3}\left(\omega_{k}^{\prime}\right)}{\omega_{k^{\prime}}}\right]$

III. The spectral function.

The problem now is to transform $f_{E}\left(\vec{q}^{2}\right)$ and $f_{\mathbb{M}}\left(\vec{q}^{2}\right)$ to a spectral representation. To do this it is useful to have the following integral representations of the terms appearing in $R_{i}\left(w_{K}\right)$. These representations are derived by considering the Cauchy integral in the complex $K$ plane with cuts from $i \mu$ to $i \infty$ and deforming the contours to run along the cuts paying attention to the proper sign of the square root along each side of the cuts. 


$$
h_{i}\left(\omega_{k}\right)=\int_{\mu^{2}}^{\infty} \frac{e_{i}\left(\alpha^{2}\right) d \alpha^{2}}{\alpha^{2}+k^{2}}
$$

i

1

2

3

4

$$
\frac{h_{i}\left(\omega_{k}\right)}{\frac{1}{w_{k}+w_{R}}}
$$$$
\frac{1}{\left(w_{k}+w_{R}\right)^{2}}
$$

$$
\frac{1}{w_{k}\left(w_{k}+w_{R}\right)}
$$

$$
\frac{1}{w_{k}\left(w_{k}+w_{R}\right)^{2}}
$$

$$
\frac{1}{\pi} \frac{\varphi_{i}\left(\alpha^{2}\right)}{\frac{\sqrt{\alpha^{2}-\mu^{2}}}{\alpha^{2}-\mu^{2}+\omega_{R^{2}}}}
$$$$
\frac{2 \omega_{R}}{\pi} \frac{\sqrt{\alpha^{2}-\mu^{2}}}{\left(\alpha^{2}-\mu^{2}+\omega_{R}^{2}\right)^{2}}
$$$$
\frac{1}{\pi} \frac{1}{\sqrt{\alpha^{2}-\mu^{2}}} \frac{\omega_{R}}{\omega_{R}^{2}+\alpha^{2}-\mu^{2}}
$$$$
\frac{1}{\pi} \frac{1}{\sqrt{\alpha^{2}-\mu^{2}}} \frac{\omega_{R}^{2}+\mu^{2}-\alpha^{2}}{\left(\omega_{R}^{2}-\mu^{2}+\alpha^{2}\right)^{2}}
$$

The contributions from the resonance will be calculated first and the contributions from the Born terms can easily be found by the substitutions

$$
\begin{aligned}
\text { in } f_{E}\left(q^{2}\right): & w_{R} \rightarrow 0 & -2 a^{2} \rightarrow f^{2} \\
f_{M}\left(q^{2}\right): & w_{R} \rightarrow 0 & a^{2} \rightarrow f^{2}
\end{aligned}
$$

One may note in passing that since the contributions to $f_{E}\left(\vec{q}^{2}\right)$ from the Born term and resonance enter as $f^{2}$ and $-2 a^{2}$, and since $a^{2}=\frac{4}{q} f^{2}$, this term will be quite sensitive to assumptions about the resonance whereas $f_{M}\left(\vec{q}^{2}\right)$ will not be.

One now proceeds as follows:

a) Notice first that

$$
\frac{1}{k^{2}-k^{\prime 2}}\left[\frac{1}{\alpha^{2}+k^{2}}-\frac{1}{\alpha^{2}+k^{\prime 2}}\right]=-\frac{1}{\left(\alpha^{2}+k^{2}\right)\left(\alpha^{2}+k^{\prime 2}\right)}
$$


b) It is convenient to forget about the cut-off at this time. It will be needed further on to justify a partial integration but the final spectral functions, since they give the low mass behaviour, do not depend on the cut-off.

c) Introduce a charge of variables

$$
\begin{aligned}
\vec{k}^{\prime} & =-\left(\vec{v}+\frac{\vec{u}}{2}\right) \\
\vec{k} & =\vec{v}-\frac{\vec{u}}{2} \\
d^{3} k d^{3} k^{\prime} & =d^{3} v d^{3} u
\end{aligned}
$$

This gives a $\delta(\vec{u}-\vec{q})$ and the $\vec{u}$ integration can be performed.

d) Introduce the Feynman parametrization

$$
\frac{1}{a b}=\int_{0}^{1} d x \frac{1}{[a x+b(1-x)]^{2}}
$$

and make a second charge of variables to $\vec{t}=\vec{V}-\overrightarrow{\frac{q}{2}}(1-2 x)$. The denominators become independent of angle and the angular integrations in the numerators can be carried out. Note that

$$
\begin{aligned}
& \int d \Omega_{t}(\text { terms odd in } \vec{t})=0 \\
& \int d \Omega_{t}(\vec{a} \cdot \vec{t})(\vec{b} \cdot \vec{t})=\frac{4 \pi}{3} t^{2}(\vec{a} \cdot \vec{b})
\end{aligned}
$$

The results of these four steps are

$$
\begin{aligned}
& f_{E}^{R}\left(\vec{q}^{2}\right)=-\frac{8 e \tau_{3}}{\pi}\left(\frac{a^{2}}{\mu}\right)^{1} \int_{0}^{\infty} d x \int_{\mu^{2}}^{\infty} d x^{2} \int_{0}^{\infty} \frac{t^{2} d t}{\left[t^{2}+A^{2}\right]^{2}}\left[\left(t^{2}-\vec{q}^{2} x(1-x)\right) p_{1}\left(\alpha^{2}\right)-\frac{1}{2 M}\left\{\left(t^{2}-\vec{q}^{2} k(1-x)\right)^{2}+\frac{1}{3} t^{2} \vec{q}^{2}(1-2 x)^{2}\right\} \rho_{2}\left(\alpha^{2}\right)\right] \\
& F_{M}^{R}\left(\vec{q}^{2}\right)=\frac{4 e \pi_{3}}{3 \pi}\left(\frac{a}{\mu}\right)^{2} \int_{0}^{1} d x \int_{\mu^{2}}^{\infty} d \alpha_{0}^{2} \int_{0}^{\infty} \frac{t^{2} d t}{\left[t^{2}+A_{1}^{2}\right]^{2}}\left[t^{2} \rho_{3}\left(\alpha^{2}\right)-\frac{1}{2 M} t^{2}\left(t^{2}-\vec{q}^{2} x(1-x)\right) \rho_{4}\left(\alpha^{2}\right)\right]
\end{aligned}
$$

where $A^{2}=\vec{q}^{2} x(1-x)+x^{2}$. 
e) The $\vec{q}^{2}$ dependence must now be removed from the numerators. To do this use

$$
\begin{aligned}
t^{2}-\vec{q}^{2} \times(1-x) & =2 t^{2}+\alpha^{2}-\left(t^{2}+A^{2}\right) \\
\vec{q}^{2} & =\frac{1}{x(1-x)}\left[\left(t^{2}+A^{2}\right)-t^{2}-\alpha^{2}\right]
\end{aligned}
$$

When this is done, one finds one term in $f_{E}^{R}\left(\vec{q}^{2}\right)$ which has no $\vec{q}^{2}$ dependence. Thus one must write

$$
F_{E}^{R}\left(\vec{q}^{2}\right)=F_{E}^{R}(\infty)+{\widetilde{F_{E}}}_{E}\left(\vec{q}^{2}\right)
$$

and including the Born term one has

$$
f_{E}(\infty)=-\frac{2 e \tau_{3}}{3 \pi M} \Lambda^{3}\left[\left(\frac{f}{\mu}\right)^{2}-2\left(\frac{a}{\mu}\right)^{2}\right]
$$

where an upper limit $\Lambda$ has been put in the $t$ integration. Since this term corresponds to $f_{E}\left(\vec{q}^{2}=\infty\right)$ one can say nothing about it on the basis of the static theory. It can be removed by relaxing the condition that equation (1) also gives the correct charge and performing one subtraction

$$
F_{E}\left(q^{2}\right)-F_{E}(0)=-\frac{q^{2}}{\theta_{\pi}} \int_{4 \mu^{2}}^{\infty} \frac{G_{E}\left(\sigma^{2}\right) d \sigma^{2}}{\sigma^{2}\left(\sigma^{2}+q^{2}\right)}
$$

Therefore it is the function $\widetilde{f}_{E}^{R}\left(q^{2}\right)$ which shall be investigated further.

$$
\begin{aligned}
\text { The remaining terms in } & \frac{1}{\left[t^{2}+A^{2}\right]^{2}} \quad \text { are removed by a } \\
\text { partial integration setting } & d r=\frac{t d t}{\left[t^{2}+A^{2}\right]^{2}} \quad s=[\text { what is left]. } \\
r & =-\frac{1}{2\left(t^{2}+A^{2}\right)}
\end{aligned}
$$


Note two things :

1) Since there is a cut-off $\left.5 r\right|_{0} ^{\infty}=0$

2) Since one is interested in the spectral function for low mass behaviour, the derivative of the cut-off function may be set equal to zero. This results in

$$
\begin{aligned}
& F_{H}^{R}\left(\vec{q}^{2}\right)=\frac{2 e \tau_{3}}{3 \pi}\left(\frac{a}{\mu}\right)^{2} \int_{0}^{1} d x \int_{\mu^{2}}^{\infty} d \alpha^{2} \int_{0}^{\infty} \frac{d t}{t^{2}+\dot{A}^{2}}\left[3 t^{2} \rho_{3}\left(\alpha^{2}\right)-\frac{1}{2 M}\left(8 t^{4}+3 t^{2} \alpha^{2}\right) \rho_{4}\left(\alpha^{2}\right)\right] \\
& \tilde{f}_{E}^{R}\left(\vec{q}^{2}\right)=-\frac{4 e \tau_{3}}{\pi}\left(\frac{a}{\mu}\right)^{2} \int_{0}^{1} d x \int_{\mu^{2}}^{\infty} \int_{0}^{\infty} d \alpha^{2} \frac{d t}{t^{2}+\beta^{2}}\left[\left(4 t^{2}+\alpha^{2}\right) \rho_{1}\left(\alpha^{2}\right)-\frac{1}{2 M}\left\{\left(2 t^{2}+\alpha^{2}\right)\left(6 t^{2}+\alpha^{2}\right)-\frac{t^{2}\left(t^{2}+\alpha^{2}\right)(1-2 x)^{2}}{x(1-x)}\right\} \rho_{2}\left(\alpha^{2}\right)\right] \\
& t^{2}+A^{2}=t^{2}+\alpha^{2}+\vec{q}^{2} \times(1-x) \\
& =x(1-x)\left[\vec{q}^{2}+\frac{t^{2}+\alpha^{2}}{x(1-x)}\right]
\end{aligned}
$$

One can write

$$
\frac{1}{\vec{q}^{2}+\frac{t^{2}+\alpha^{2}}{x(1-x)}}=\int_{4 p^{2}}^{\infty} \frac{d \sigma^{2} \delta\left(\sigma^{2}-\frac{t^{2}+\alpha^{2}}{x(1-x)}\right)}{\vec{q}^{2}+\sigma^{2}}
$$

where the lower limit comes from the fact that the minimum value of

$$
\begin{array}{ll}
\frac{t^{2}+\alpha^{2}}{x(1-x)} & 0 \leq t \leq \infty \\
& \mu^{2} \leq \alpha^{2} \leq \infty \\
& 0 \leq x \leq 1
\end{array} \quad \text { is } 4 \mu^{2}
$$


Putting this in and interchanging limits of integration one is left with an integrand which is just the spectral function *)

$$
\begin{aligned}
& g_{M}^{R}\left(\sigma^{2}\right)= \frac{2 e \tau_{3}}{3}\left(\frac{a}{\mu}\right)^{2} \int_{0}^{1} d x \int_{\mu^{2}}^{\infty} d \alpha^{2} \int_{0}^{\infty} d t \delta\left(t^{2}+\alpha^{2}-\sigma^{2} x(1-x)\right)\left[3 t^{2} \rho_{3}\left(\alpha^{2}\right)-\frac{1}{2 M}\left(8 t^{4}+3 t^{2} \alpha^{2}\right) \rho_{4}\left(\alpha^{2}\right)\right] \\
& g_{E}^{R}\left(\sigma^{2}\right)=-4 e \tau_{3}\left(\frac{a}{\mu}\right)^{2} \int_{0}^{1} d x \int_{\mu^{2}}^{\infty} d \alpha^{2} \int_{0}^{\infty} d t \delta\left(t^{2}+\alpha^{2}-\sigma^{2} x(1-x)\right)\left[\left(4 t^{2}+\alpha^{2}\right) \rho_{1}\left(\alpha^{2}\right)\right. \\
&\left.-\frac{1}{2 M}\left\{\left(2 t^{2}+\alpha^{2}\right)\left(6 t^{2}+\alpha^{2}\right)-\frac{t^{2}\left(t^{2}+\alpha^{2}\right)(1-2 x)^{2}}{x(1-x)}\right\} \rho_{2}\left(\alpha^{2}\right)\right]
\end{aligned}
$$

h) Now note the following : for the $\delta$ function to vanish one must have $\sigma^{2} \times(1-x)-\alpha^{2} \geqslant 0$

1) Since $\sigma^{2} x(1-x) \leq \frac{\sigma^{2}}{4}$ this implies $\alpha^{2} \leq \frac{\sigma^{2}}{4}$

2) For given $\alpha^{2}, \quad x=\frac{1}{2}\left[1 \pm\left(1-\frac{4 \alpha^{2}}{\sigma^{2}}\right)^{1 / 2}\right]$ is the region of vanishing of the argument of the $\delta$ function.

The ranges of integration thus reduce to $\left.\int_{\mu^{2}}^{\sigma^{2} / 4} d \alpha^{2} \int_{\frac{1}{2}\left[1-\left(1-\frac{4 \alpha^{2}}{\sigma^{2}}\right)^{1 / 2}\right\rceil}^{\frac{1}{2}\left[1+\left(1-\frac{4 \alpha^{2}}{\sigma^{2}}\right)^{1 / 2}\right\rceil} \int_{0}^{\infty} \frac{d t}{2\left(\sigma^{2} x(1-x)-\alpha^{2}\right)^{1 / 2}} \delta\left(t-\mid \sigma^{2} x(1-x)-\alpha^{2}\right]\right)[\ldots .$.

*) Note $\delta(a x)=\frac{1}{a} \delta(x)$ and $\delta\left(t^{2}-a^{2}\right)=\frac{1}{2 a} \delta(t-a)$ for $t>0$ 
The $\mathrm{x}$ integrations are easy to carry out since the square root in the integrand vanishes at the end points. This results in

$$
\begin{aligned}
& g_{H}^{R}\left(\sigma^{2}\right)=\frac{\pi}{\sigma} e \tau_{3}\left(\frac{a}{\mu}\right)^{2} \int_{\mu^{2}}^{\frac{\sigma^{2}}{4}} d \alpha^{2}\left(\frac{\sigma^{2}}{4}-\alpha^{2}\right)\left[\frac{1}{2} \rho_{3}\left(\alpha^{2}\right)-\frac{1}{4 M}\left(\frac{\sigma^{2}}{2}-\alpha^{2}\right) \rho_{4}\left(\alpha^{2}\right)\right] \\
& g_{E}^{R}\left(\sigma^{2}\right)=-\frac{2 \pi e \tau_{3}}{\sigma}\left(\frac{a}{\mu}\right)^{2} \int_{\mu^{2}}^{2} d \alpha^{2}\left(\frac{\sigma^{2}}{2}-\alpha^{2}\right)\left[\theta_{1}\left(\alpha^{2}\right)-\frac{1}{2 M}\left(\frac{\sigma^{2}}{2}-\alpha^{2}\right) \rho_{2}\left(\alpha^{2}\right)\right]
\end{aligned}
$$

The remaining integrations are elementary and yield, after the Born terms are included

$$
\begin{aligned}
& q_{H}\left(\sigma^{2}\right)=e \tau_{3}\left(\frac{q \pi^{2}}{\sigma}\right)\left[\frac{\pi}{2}\left(\frac{f}{\mu}\right)^{2}+\left(\frac{a}{\mu}\right)^{2}\left(\frac{-u_{k}}{q_{\pi}}+\frac{q_{\pi^{2}}^{2}+\omega_{k}^{2}}{q^{2}} \tan ^{-1} \frac{q \pi}{\omega_{R}}\right)-\frac{q \pi}{M}\left\{\left(\frac{f}{\mu}\right)^{2}\left(\frac{2 q^{2}+\mu^{2}}{q \pi^{2}}\right)\right.\right. \\
& \left.\left.+\left(\frac{1}{3}\left(\frac{f}{\mu}\right)^{2}-\frac{2}{3}\left(\frac{a}{\mu}\right)^{2}\right)+\left(\frac{a}{\mu}\right)^{2}\left(\frac{3 q^{2} \pi^{2}+2 \omega_{R}^{2}+\mu^{2}}{q \pi^{2}}\right)\left(1-\frac{\omega_{R}}{q_{\pi}} \tan ^{-1} \frac{q_{\pi}}{\omega_{R}}\right)\right\}\right]
\end{aligned}
$$

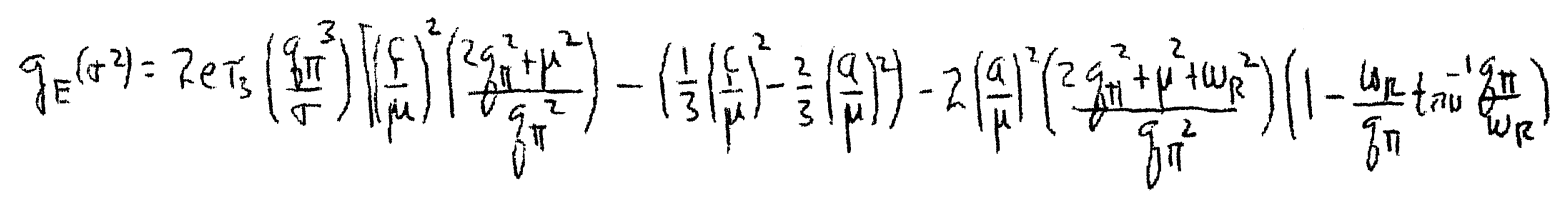

$$
\begin{aligned}
& -\frac{q \pi}{2 \mu}\left\{\left(\frac{\xi}{\mu}\right)^{2}\left(\frac{\pi}{2}\right)\left(\frac{2 q_{\pi}^{2}+\mu^{2}}{g^{2}}\right)^{2}-2\left(\frac{a}{\mu}\right)^{2}\left[-\frac{2 \omega_{R}}{3 q_{\pi}}\left(\frac{11 q_{\pi}^{2}+6 \mu^{2}+5 \omega_{k}^{2}}{q \pi^{2}+\omega_{k}^{2}}\right)+\right.\right. \\
& \left.\left.\left.\frac{\left(2 q^{2}+\mu^{2}+\omega_{R}^{2}\right)\left(2 q^{2}+\mu^{2}+5 \omega_{R}^{2}\right)}{q \pi^{2}\left(q^{2}+\omega_{R}^{2}\right)}\left[\frac{q^{2}+\omega_{R}^{2}}{q \pi^{2}} \tan ^{-1} \frac{q_{\pi}}{w_{R}}-\frac{\omega_{R}}{q_{\pi}}\right]\right]\right\}\right]
\end{aligned}
$$

where

$$
9 \pi^{2}=\frac{\sigma^{2}}{4}-\mu^{2}
$$


The $S$ matrix for a single scattering in an external electromagnetic field is given by ${ }^{*}$ )

$$
\left\langle p^{\prime}|s| p\right\rangle=-\frac{1}{\Omega} \bar{u}\left(p^{\prime}\right) \Gamma_{\mu}\left(q^{2}\right) u(p) A_{\mu}^{\text {ent }}(q)
$$

where $p^{\prime}=p+q, \Omega$ is the volume of the normalization box, and $A^{\text {ext }}(q)$ is the Fourier transformof $A^{\text {ext }}(x)$.

Now

$$
\Gamma_{\mu}\left(q^{2}\right)=F_{E}\left(q^{2}\right) \gamma_{\mu}-F_{M}\left(q^{2}\right) \sigma_{\mu \nu} q_{\nu}
$$

Making the non-relativistic reduction to order $\frac{1}{\mathbb{M}}$ by taking

$$
u(p)=\left(\begin{array}{c}
1 \\
\frac{\vec{T}, \vec{p}}{2 M}
\end{array}\right)
$$

one finds (in the Breit frame-note again $\vec{q}^{2}=q^{2}$ )

$$
\begin{aligned}
& \left\langle\frac{\vec{q}}{2}|s|-\frac{\vec{q}}{2}\right\rangle=-\frac{(2 \pi)^{4} i}{\Omega} \delta^{4}\left(p^{\prime}-p-q\right)\left[\left(F_{E}\left(g^{2}\right)-\frac{q^{2}}{2 H} F_{M}\left(q^{2}\right)\right) \phi-i \vec{\sigma}(\vec{g} \wedge \vec{a})\left(F_{M}\left(q^{2}\right)+\frac{F_{E}\left(q^{2}\right)}{2 M^{0}}\right)\right] \\
& A_{\mu}^{\operatorname{lnt}}(x)=a_{\mu} e^{i q x} \\
& a_{\mu}=(\vec{a}, i \phi)
\end{aligned}
$$

The above is the full $S$ matrix to first order in the external field.

*) $\quad \gamma_{\mu} \gamma_{\nu}+\gamma_{\nu} \gamma_{\mu}=2 \delta_{\mu \nu}$

$$
\begin{aligned}
& A_{\mu}=(\vec{A}, i \phi) \\
& P_{\mu}=(\vec{p}, i E)
\end{aligned}
$$


Going now to an interaction representation where the time dependence is given by the full meson nucleon Hamiltonian and $\mathrm{H}_{1}=\mathrm{H}$ electromagnetic, one has

$$
\begin{aligned}
& \left\langle\frac{\vec{q}}{2}|S|-\frac{\vec{q}}{r_{2}}\right\rangle=-1\left\langle\frac{\vec{q}}{2}\left|\int H_{1}^{e x}(x) d 4 \times\right|-\frac{\vec{q}}{q_{2}}\right\rangle \\
& =-2 \pi i \delta\left(E_{f}-E_{i}\right)\left\langle\frac{\vec{q}}{2}\left|\int 1 H_{1}^{e+}(x) d^{3} x\right|-\vec{g} \vec{q}_{2}\right\rangle \\
& =-2 \pi i S\left(E_{F}-E_{i}\right) \omega
\end{aligned}
$$

Therefore, since $\quad \frac{(2 \pi)^{3}}{\Omega} \delta\left(\vec{p}^{\prime}-\vec{p}-\vec{q}\right)=1$

$$
\begin{aligned}
& \omega=f_{E}\left(q^{2}\right) \phi-i \sigma \cdot\left(\overrightarrow{q_{A}} \vec{a}\right) F_{M}\left(q^{2}\right) \\
& F_{E}\left(q^{2}\right)=F_{E}\left(q^{2}\right)-\frac{q^{2}}{2 H} F_{M}\left(q^{2}\right) \\
& F_{M}\left(q^{2}\right)=F_{M}\left(q^{2}\right)+\frac{F_{E}\left(q^{2}\right)}{2 M}
\end{aligned}
$$

For the second equation, one can add the corresponding spectral functions, however, for the first equation one must write

$$
\begin{aligned}
& F_{E}\left(q^{2}\right)=\frac{1}{\pi} \int_{4 \mu^{2}}^{\infty} d \sigma^{2}\left[\frac{G E\left(\sigma^{2}\right)}{\sigma^{2}+q^{2}}-\frac{q^{2}}{2 \mu} \frac{G M\left(\sigma^{2}\right)}{\sigma^{2}+q^{2}}\right] \\
& =F_{E}(\infty)+\hat{F}_{E}\left(q^{2}\right) \\
& f_{E}(\infty)=-\frac{1}{2 M \pi} \int_{4 \mu^{2}}^{\infty} G_{M}\left(\sigma^{2}\right) d \sigma^{2}
\end{aligned}
$$

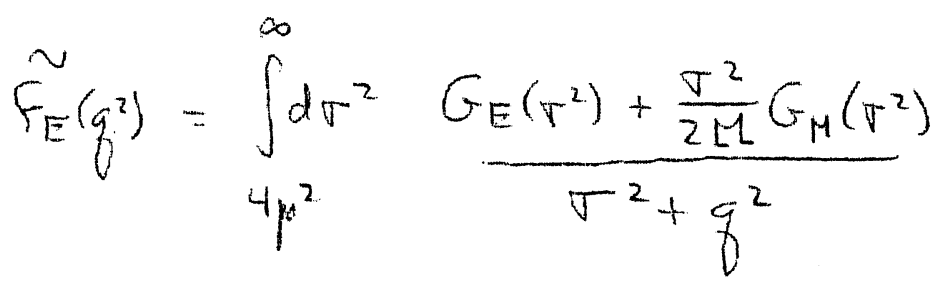


Thus if one uses a superscript (1) to indicate terms up through $\frac{1}{\mathbb{M}}$ and a (0) to indicate the static limit, one finds for the static theorł for $G_{E}^{(1)}\left(\sigma^{2}\right)$ and $G_{\mathbb{N}}^{(1)}\left(\sigma^{2}\right)$

$$
\begin{aligned}
& G_{M}^{(1)}\left(\sigma^{2}\right)_{S . T}=g_{M}^{(1)}\left(\sigma^{2}\right)-\frac{g_{E}^{(0)}\left(\sigma^{2}\right)}{2 M} \\
& G_{E}^{(1)}\left(\sigma^{2}\right)_{S . T .}=g_{E}^{(1)}\left(\sigma^{2}\right)-\frac{\sigma^{2}}{2 M} g_{M}^{(0)}\left(\sigma^{2}\right)
\end{aligned}
$$

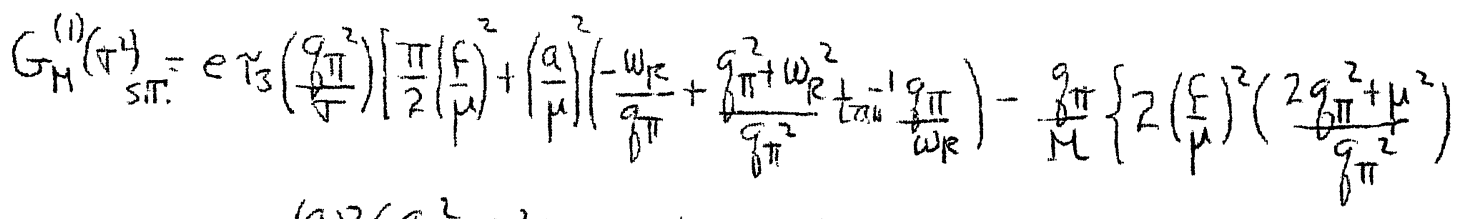

$$
\begin{aligned}
& \left.\left.-\left(\frac{a}{\mu}\right)^{2}\left(\frac{q \pi^{2}+\mu^{2}}{q \pi^{2}}\right)\left(1-\frac{w_{R}}{q \pi} \tan ^{-1} \frac{q \pi}{w_{R}}\right)\right\}\right]
\end{aligned}
$$

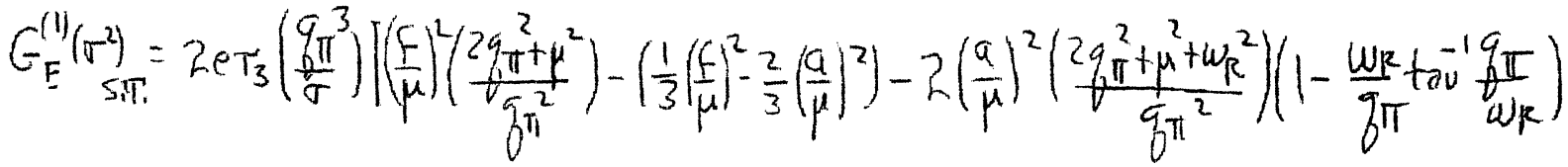

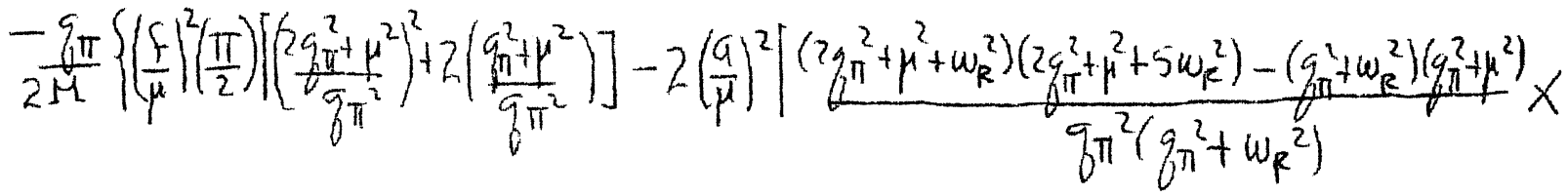

$$
\begin{aligned}
& \left.\left.\left.\times\left(\frac{q \pi^{2}+\omega_{R}^{2}}{q \pi^{2}} \tan \frac{-1 q \pi}{\omega_{R}}-\frac{\omega_{R}}{q \pi}\right)-\frac{2}{3} \frac{\omega_{R}}{q \pi}\left(\frac{11 q^{2}+6 \mu^{2}+5 \omega_{R}^{2}}{q \pi^{2}+\omega_{R}^{2}}\right)\right]\right\}\right]
\end{aligned}
$$

The corresponding equations of dispersion theory obtained by expanding equations 15.1 to 15.7 of reference 1) to order $\frac{1}{\bar{M}}$ are

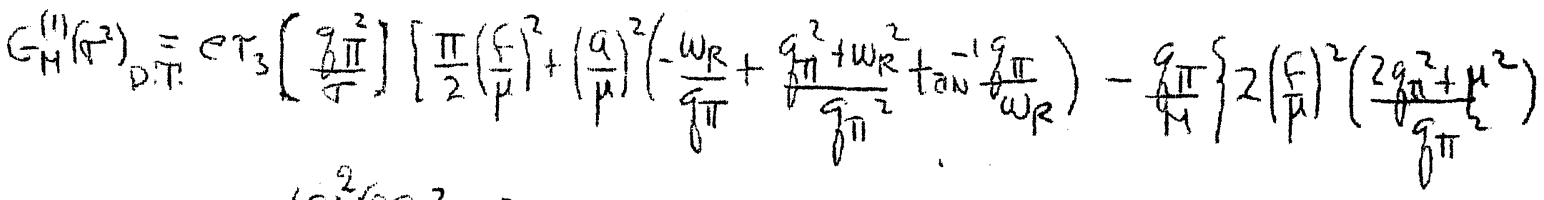

$$
\begin{aligned}
& \left.\left.-\left(\frac{a}{\mu}\right)\left(\frac{2 g^{2}+\mu^{2}+\omega_{R}^{2}}{g^{2}}\right)\left(1-\frac{\omega_{R}}{\sigma^{2}} \tan ^{-1} \frac{q \pi}{\omega_{R}}\right)\right\}\right]
\end{aligned}
$$




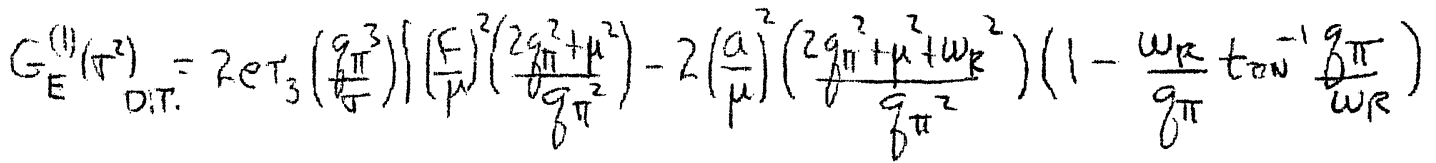

$$
\begin{aligned}
& -\frac{q \pi}{2 M}\left\{\left(\frac{f}{\mu}\right) \frac{\pi}{2}\left[\left(\frac{2 g^{2}+\mu^{2}}{g_{j}^{2}}\right)^{2}+2\left(\frac{q^{2}+\mu^{2}}{q \pi^{2}}\right)\right]-2\left(\frac{a}{\mu}\right)^{2}\left[\frac{\left(2 q^{2}+\omega_{R}^{2}+\mu^{2}\right)^{2}-\left(q_{n}^{2}+3 \omega_{R}^{2}\right)\left(q^{2}+\mu^{2}\right)}{q \pi^{2}\left(q \pi^{2}+\omega_{R}^{2}\right)} x\right.\right. \\
& \left.\left.\left.\times\left(\frac{q \pi^{2}+\omega_{R}^{2}}{g \pi^{2}} \tan ^{-1} \frac{q \pi}{\omega_{R}}-\frac{w_{R}}{9 \pi}\right)+\frac{2 \omega_{R}}{9 \pi}\left(\frac{9 \pi^{2}+\mu^{2}}{q \pi^{2}+\omega_{R}^{2}}\right)\right]\right\}\right]
\end{aligned}
$$

In figures 1 and 2 are plotted $G_{\mathbb{N}, \mathbb{E}}^{(0)}\left(\sigma^{2}\right)_{S \cdot T}, G_{\mathbb{M}, E}^{(1)}\left(\sigma^{2}\right)_{S \cdot T \cdot 9}$ $G_{\mathbb{M}, E}^{(1)}\left(\sigma^{2}\right)$ D.T.' and also $G_{\mathbb{M}, \mathbb{T}}\left(\sigma^{2}\right)$ D.T. obtained from the equations of reference 1).

V. Summary and discussion of results.

To summarize the results :

a) One can calculate spectral functions within the framework of the static theory by a direct transformation of the form factors.

b) The results obtained agree with the static limit of the results obtained from dispersion theory (except for the term proportional to $\quad \frac{1}{3}\left(\frac{f}{\mu}\right)^{2}-\frac{2}{3}\left(\frac{a}{\mu}\right)^{2} \quad$ in $g_{E}\left(\sigma^{2}\right)$ in the static theory which must come from the neglect of pair effects, it happens to be almost zero). 
c) The $\frac{1}{\bar{M}}$ corrections coming solely from the addition of the kinematics of nucleon recoil are very nearly equal to $_{\text {to }}$ the corresponding corrections in the dispersion theory and the fact that they are so large raises serious doubts as to the applicability of the static model to the study of the electronagnetic structure of the nucleon.

The physics of the situation is this - the nucleon likes to emit and absorb P-wave mesons, and indeed, if it is infinitely heavy can only do so. After the meson picks up a photon from the external fields, it is no longer in a $P$ state relative to the nucleon, but has a great probability of being in a higher angular momentum state. If now one allows the nucleon to move and hence interact with mesons of higher angular momentum, even though the interaction is small, the amplitude of such a wave may be large so that the result may compete with P-wave absorption. This is what was found above.

\section{Acknowledgements}

The author would like to express his great indebtness to Professor S. Fubini for suggesting the problem and for his assistance throughout. He would also like to acknowledge a stimulating correspondence with Professor S. Drell and to thank Professor B. Ferretti and the Theoretical Study Division at CERN for the hospitality extended to him during his stay

\footnotetext{
*) Notice that the $\frac{1}{\mathbb{M}}$ Born terms are given exactly.
} 
1) G.F. Chew, R. Karplus, S. Gasiorowicz and F. Zachariasen, Phys.Rev. 110, 265 (1958).

2) P. Federbusch, M. Goldberger and S. Treiman (to be published).

3) S. Drell, Proceedings of the 8th. Annual Conference on High Energy Physics, Geneva, 1958.

4) G. Salzman, Phys, Rev. 99, 973 (1955),

G. Salzman, Phys.Rev. 105, 1076 (1957),

H. Niyazawa, Phys.Rev. 101, 1564 (1956),

S. Treiman and R.G. Sachs, Phys.Rev. 103, 435 (1956),

F. Zachariasen, Phys.Rev. 102, 295 (1956),

S. Fubini, Nuovo Cimento 3, 1425 (1956).

5) B. Bosco, S. Fubini and A. anghellini (to be published).

6) M. Cini and S. Fubini, Nuovo Cimento 3, 764 (1956).

\section{FIGURE CAPTIONS}

Fig. I

The spectral functions for the magnetic form factor of the nucleon as calculated from dispersion theory (D.T.) and in the static theory including $\frac{1}{\sqrt{M}}$ corrections coming from the kinematics of nucleon recoil (S.T.). (O) indicates the static limit and (1) indicates terms up through $\frac{1}{M}$.

Fig. II

Same functions as in Fig. 1 for the charge form factor of the nucleons. 


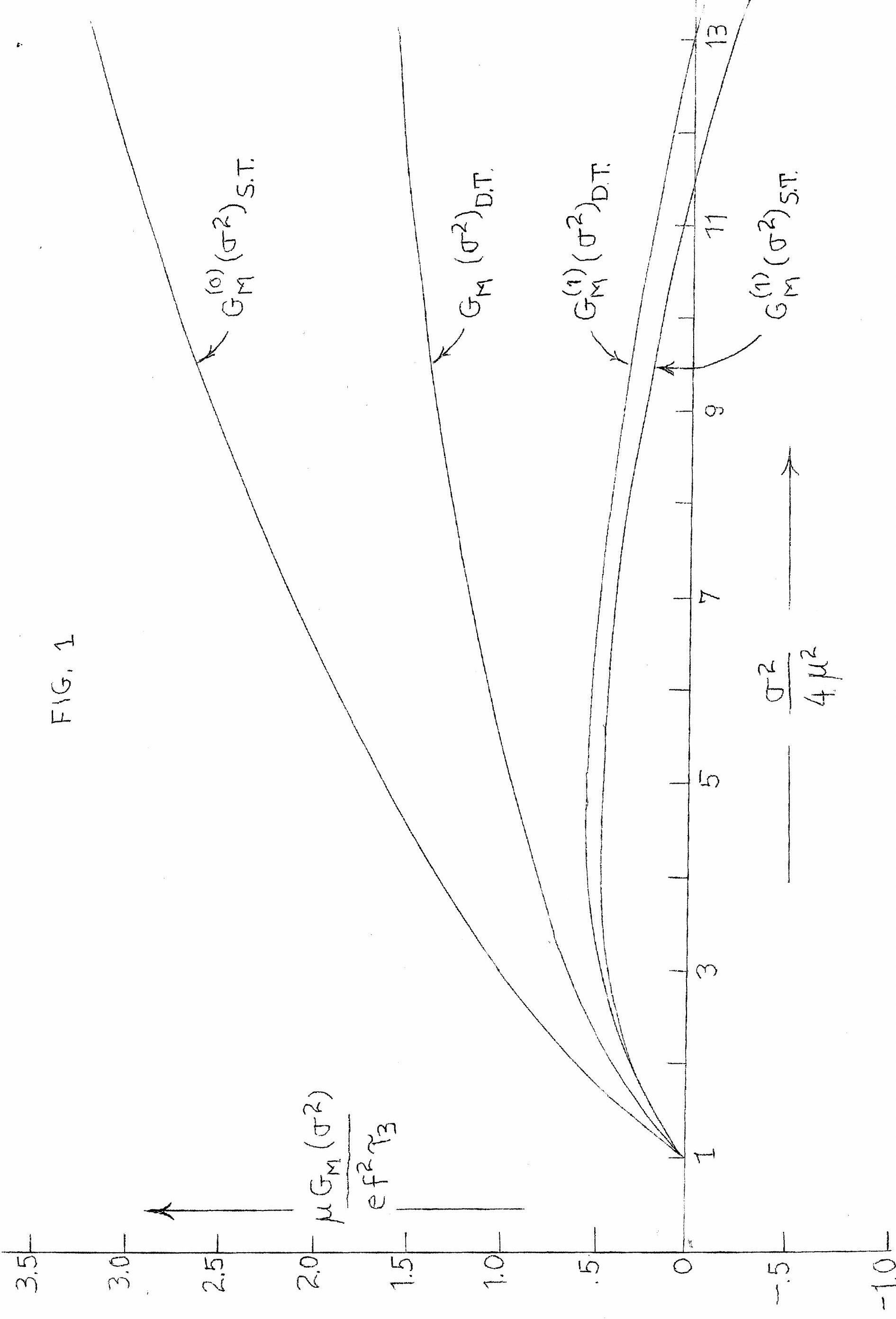




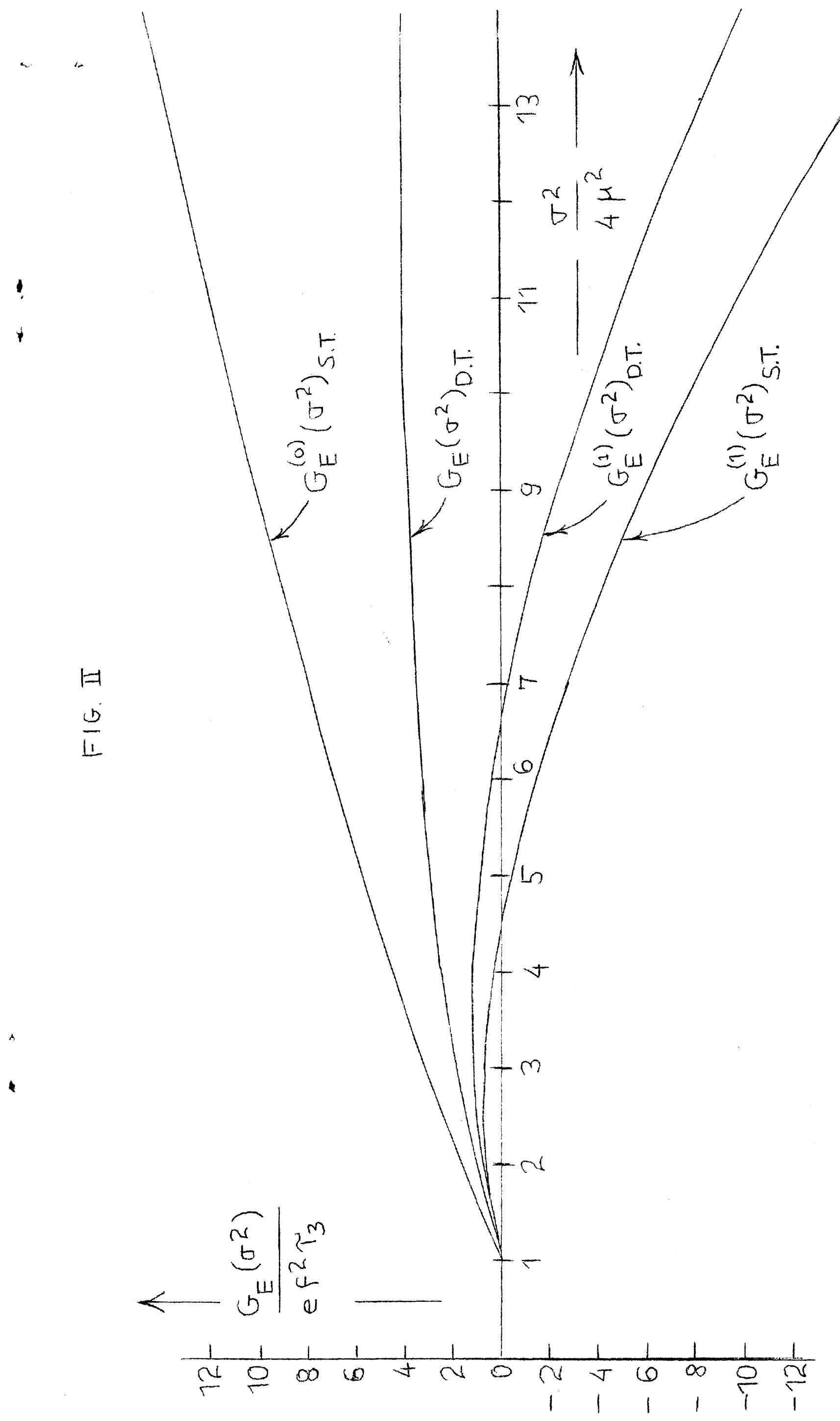

